\title{
Towards reducing behavioral risk factors of non-communicable diseases among adolescents: protocol for a school-based health education program in Bangladesh
}

Marium Salwa ${ }^{1 *}$ (D), Md Atiqul Haque², Md Khalequzzaman², Mohammad Abdullah Al Mamun², Mahfuzur Rahman Bhuiyan ${ }^{3}$ and Sohel Reza Choudhury ${ }^{3}$

\begin{abstract}
Background: Developing strategies aimed at reducing behavioral risk factors and hence the prevalence of noncommunicable diseases (NCDs) is a major challenge to the policy makers today. Like the same age group worldwide, the prevalence of obesity, unhealthy dietary habit, physical inactivity, smoking and alcohol intake is high among the adolescents of Bangladesh. Studies showed promising results of an early intervention at adolescent age in reducing the likelihood of NCDs at adult age. So, this study is designed to implement a behavior change intervention and evaluate the effectiveness of the intervention in reducing the behavioral risk factors of NCDs among the adolescents of Bangladesh.
\end{abstract}

Methods: A before-after designed intervention study will be conducted in two randomly selected secondary schoolsone will be selected randomly as intervention school and the another as control school. A baseline survey will be conducted among the students of both schools by a pre-tested questionnaire to attain their current status of knowledge, attitude and practices related to NCDs. Afterward, students will be enrolled in the intervention group who will meet the eligibility criteria from the intervention school. The intervention will be given through a health promotion session to a group of students, not more than 25 at a time, by trained facilitators. A post-intervention end line survey will be conducted among all the participants from both schools using the same questionnaire 3 months after the baseline survey.

Discussion: An intervention has been developed based on some principals of two psychosocial theory- Motivational Interview and Social Cognitive Theory. Emphasis will be given on motivating the adolescents towards a healthy lifestyle, supporting self-efficacy to be changed, guiding self-regulatory ways along with facilitating desired changing process by empowering them with choices about the preventive measures of NCDs. This intervention is expected to increase awareness by equipping the adolescents with specific knowledge and skills and thus, facilitate an eventual change in their practiced risk behaviors. Besides, this intervention will address multiple behaviors at a time, and will be delivered to a group of adolescents, to attain the cost-effectiveness and thereby making it more realistic in the resource-poor context of Bangladesh.

Trial registration: ClinicalTrials.gov NCT03975335, registered on 01.06.2019. Retrospectively registered.

Keywords: Behavioral intervention, Health education, Adolescent, NCD risk factors, Bangladesh

\footnotetext{
* Correspondence: mariumsalwa@gmail.com

${ }^{1}$ Chi Research \& Infotec Ltd, Dhaka, Bangladesh

Full list of author information is available at the end of the article
}

(c) The Author(s). 2019 Open Access This article is distributed under the terms of the Creative Commons Attribution 4.0 International License (http://creativecommons.org/licenses/by/4.0/), which permits unrestricted use, distribution, and reproduction in any medium, provided you give appropriate credit to the original author(s) and the source, provide a link to the Creative Commons license, and indicate if changes were made. The Creative Commons Public Domain Dedication waiver (http://creativecommons.org/publicdomain/zero/1.0/) applies to the data made available in this article, unless otherwise stated. 


\section{Background}

Towards addressing the non-communicable diseases (NCDs) epidemic in Bangladesh, effective measures are needed to stifle its emergence. In the case of NCDs, it is useful to start the prevention process at the adolescent stage [1] since most of the behavior related risk factors of NCDs begin to demonstrate at this age [2]. Targeting adolescents also has the additional benefit of reducing the life-time cost of healthcare by addressing the behaviors at the outset $[3,4]$.

Adolescents of Bangladesh, like the rest of the world, are exposed to significant amount of NCDs risk factors such as obesity [5], less fruit and vegetable intake [6], physical inactivity [7], smoking [8] etc. Given modifiable risk factors are prevalent in large numbers, intervention that aims to change behavior can be an effective method of halting the growth of NCDs. Several theories on how human behavior is shaped have been discussed and evaluated over the past decades or so. For example, attitude towards a behavior and subjective norm or social influence have been emphasized to influence human behavior in many theories including theory of planned behavior [9]. Meta-analysis supported the theory that there is a strong relationship between attitude and behavior, and behavioral intention plays a major role as a mediator between them [10]. Furthermore, greater attitudinal consistency is observed amongst the better informed [11]. Self-efficacy expectation or personal belief to accomplish a change in behavior described by Bandura in his Social Cognitive Theory (SCT) [12, 13] was proven to have successfully predicted behavioral intention [14, 15]. Moreover, adolescents were found in need of tremendous psychological support or motivation to shape positive behaviors [16]. Motivational Interview (MI) postulated by Rollnick \& Miller (1995) [17] showed promising results to this effect.

Understanding behavioral risk and protective factors can positively influence the development of intervention programs [18]. Theory-based intervention has proven to be an effective method in driving behavioral change. However, choosing the right intervention technique is a difficult task and needs to be experimented and evaluated in context specific settings [19, 20]. Studies suggested that the choice of a suitable theory base for an intervention should begin with identifying the problem, goal, and units of practice [18]. Also, intervention targeting multiple behavior has been proven to have greater impact than single behavior intervention [21, 22].

Considering all these aspects, an intervention has been designed to reduce NCDs related risk behaviors such as unhealthy dietary habit, physical activity and smoking among the adolescent of Bangladesh considering some of the components of MI and SCT. In this intervention, emphasis is given on evoking adolescents' internal desire to change, supporting self-efficacy to be changed, guiding self-regulatory ways along with facilitating desired changing process by empowering them with choices and skills. It is to clarify that, this study will not measure the efficacy of the theory of MI or SCT, rather the effectiveness of the proposed intervention will be evaluated which has been constructed based on some principles of the former theories. This intervention is expected to increase awareness by equipping the adolescents with specific knowledge, motivating them, and facilitating an eventual change in their practiced risk behaviors.

\section{Objectives}

\section{General objective}

To design and investigate the efficacy of a health education intervention to reduce NCD related behavioral risk factors among adolescents in institutional settings of Bangladesh.

\section{Specific objectives}

1. To conduct a baseline survey to evaluate the knowledge, attitude and practices about NCD risk behaviours such as unhealthy diet, physical inactivity, smoking etc. among the respondents.

2. To develop a health education intervention based on the baseline survey to reduce NCDs related behavioural risk factors among adolescents.

3. To apply the health education intervention to the target group through effective communication approach and tools.

4. To conduct a post interventional survey with the same questionnaire used in baseline data collection.

5. To evaluate the intervention by comparing pre and post intervention data.

\section{Methodology}

\section{Study design and setting}

A before-after designed intervention study will be conducted in two randomly selected secondary schools in a semi-urban area of Dhaka, capital of Bangladesh. One school will be selected randomly as intervention school and the other one as control school. Possibility of diffusion of intervention contents among students from one school to another will be minimized by selecting the schools at a reasonable distance.

\section{Participant recruitment and data collection}

The study will follow a fixed study design (Additional file 1). Sample size is determined based on a previous study where a mean difference of BMI was found 1.48 between intervention and non-intervention adolescent groups after 3-months of motivational interview [23]. Expecting a similar difference, calculated sample size is 274 in each group considering effect size $(E)=1.48$; 
standard deviation $(S)=5.98$; standardized effect size $(E /$ $\mathrm{S})=.25$; significance level, $\alpha$ (two-sided) $=0.05 ; \beta=1$ power $=0.2$, non-response rate $=5 \%]$. A complete list of grade-ten students will be prepared after collecting their class roll and batch number from the administrative offices of the selected schools. All the students of gradeten will be invited to participate in the study through announcement and personal contact prior to the date of data collection.

At first, a baseline survey will be conducted among the grade ten students of both schools by a pre-tested questionnaire to attain their current status of knowledge, attitude and practices related to NCDs. Then, students from the intervention school who reported at least two NCDs risk behaviors will be invited to participate in a health education session through phone call to their parents. Selected students who will present at the day of intervention will be considered having implied consent from their parents and will be enrolled in the intervention group up to the attainment of the required sample size. Enrolled students will be given intervention through health education session. Students with any kind of physical disability or limited movement will be excluded from the study. Students from the control group will receive a one-hour session on carrier guidance. Students will be kept blind about the intervention they received. After 3 months of the baseline survey, a post-intervention survey will be conducted among the participants from both intervention and control group using the same questionnaire.

\section{Data collection tool}

A self-administered questionnaire is being constructed after reviewing several survey questionnaires in this field including Global School Health surveys [24], STEPs Survey of NCD risk factors [25], a Mongolian survey questionnaire [26] etc. The questionnaire is being constructed first in English, then translated into Bangla and again back translated into English by experts to ensure the translation. Then the questionnaire will be pre-tested among a similar population and modification of the questionnaire will be done accordingly. Finalized questionnaire (Additional file 2) will be used both in pre-intervention baseline and post-intervention end line survey.

There will be four sections in the questionnaire- the first section of the questionnaire will contain questions on socio-demographic information of the participants. The second section will contain questions about knowledge, attitudes and practice related to NCDs e.g. cardiovascular diseases, stroke, diabetes, hypertension, cervical cancer, etc. Third section will cover questions on knowledge, attitudes, perceived barriers and practice related to behavioral risk factors of NCDs e.g. diet, physical activity, smoking and alcohol, and the fourth section will hold physical measurement of the respondents such as height, weight, blood pressure, pulse, waist and hip circumference etc.

\section{Outcome variables}

Following outcome variables will be measured.

\section{Knowledge}

Knowledge about NCDs and risk behaviors will be measured by 25 knowledge measuring questions scoring 54 in total. Answer to the questions will be regarded as correct or incorrect based on information from standard medical textbooks and guidelines.

\section{Attitude}

Attitude towards NCDs and its risk factors will be measured by nine attitude measuring questions in a Likert Scale.

\section{Practice}

Practice questions will be used to evaluate dietary habit, physical activity, smoking, alcohol and substance abuse.

Based on WHO recommendations and standard practices for adolescent age, the following practices will be measured in this study.

1. Dietary habit: presence of at least two of the following four habits will be accounted as having dietary risk behavior

i. Inadequate fruit consumption: Less than five servings of fruits per day

ii. Inadequate vegetable consumption: Less than five servings of vegetables per day

iii. Excessive salt consumption: Taking extra or raw salt during every meal

iv. Sugar Sweetened Beverage (SSB) consumption: Consuming SSB more than 3 days per week

2. Physical activity: Not meeting $60 \mathrm{~min}$ physical activity of moderate intensity per day will be regarded as physically inactive.

3. Smoking and alcohol: Smoking regularly in the last 30 days or exposing to passive smoking more than 3 days per week will be accounted as in risk of smoking. Any amount of alcohol intake or any substance abuse in the last 30 days will be regarded as at risk.

\section{Physical measurement Blood pressure}

Blood pressure will be measured by an automatic digital sphygmomanometer (HEM-8712, Omron, Kyoto, Japan). Students will be asked to sit down on a chair and rest their right elbow on a table. Blood pressure will be measured three times allowing at least $5 \mathrm{~min}$ interval between each measurement. The mean of second and third 
measurement will be taken into analysis. Presence of systolic blood pressure $\geq 130 \mathrm{~mm}$ of $\mathrm{Hg}$ and/or diastolic blood pressure $\geq 80 \mathrm{~mm}$ of $\mathrm{Hg}$ will be considered as having high blood pressure.

\section{Height}

Height will be measured by a portable and non-stretchable measuring tape. Students will be asked to remove their shoes and head gear, if any and stand straight on the flat floor against a wall, facing the interviewer. Their heels will be in touch of the wall. Interviewers will make sure that their eyes will be at the same level as the ear. Height will be recorded in centimeter to the nearest millimeter.

\section{Weight}

Weight will be measured by portable digital weighing machine (Medisana Bathroom Glass Scales). The machine will be placed on a flat floor. Students will be asked to remove their shoes and extra cloths, if any, and stand straight on the machine hanging their arms by their sides. Weight will be recorded in $\mathrm{kg}$.

\section{Body mass index (BMI)}

The ratio of body weight in kilogram and the height in meter square will be considered as BMI. Classification of BMI will be done as following-.

Underweight (Less than 18.5); Normal weight (18.524.9); Overweight (25.0-29.9) and Obese (More than 30.0).

\section{Waist and hip circumference}

Waist and hip circumference will be measured by a stretch-resistant measuring tape and recorded in centimeter to the nearest millimeter. Waist circumference will be measured at the midpoint between the lower margin of the last rib and the iliac crest and hip circumference will be measured at the widest part of the buttock with the tape parallel to the floor following WHO guideline [27]. For both the measurements, students will be asked to stand straight with feet close together and arms at the side. The measurement will be taken at the end of normal expiration.

\section{Data management plan}

Collected data will be entered into the pre-designed SPSS file (Version 21) and then checked, cleaned and recoded where needed. Only the authorized personnel will be able to access data to avoid any potential manipulation. Data set will be archived and only available on request once the study is over.

\section{Statistical analysis plan}

Descriptive analysis: Socio-demographic data of the participants e.g. gender, parental occupation, parental education status, family history of NCDs, family living standard will be obtained for both intervention and control school. All categorical variables will be summarized in frequency and percentages and continuous variables in mean and standard deviation. To estimate the association of these characteristics of intervention and control group, t-tests and chi-square tests will be done.

Inferential analysis: To estimate the efficacy of the intervention, before-after data will be analyzed and paired ttest and McNemar's chi-square tests will be done in this regard. Also, comparison will be done between the intervention and control group for pre and post-intervention status of knowledge, attitude and practices.

Association analysis: Binary regression analysis will be done to estimate the association between sociodemographic variables with changes in the outcome variables- knowledge, attitude, and practice for both groups. Also, to estimate the association of knowledge score and attitude with the change in self-reported practices, regression analysis will be done.

\section{Quality control measurement}

Quality of the data and adherence with the protocol will be ensured by a quality control panel consisting of four senior physicians from Bangabandhu Sheikh Mujib Medical University (BSMMU).

The facilitators to deliver the intervention will be practicing physician who will have experiences on individual patient counseling. Selected facilitators will be trained on the intervention by written dialogues addressing the sequential framework of the intervention model in a 5days workshop. Then they will have to perform dry run. Their lectures will be recorded and then scrutinized to ensure standard and similarity between the lectures. A supervisor team consisting of a psychologist, a child health specialist, a public health specialist and a nutritionist will be present during the dry run and at the actual intervention session to ensure the quality. All the sessions will be audio-recorded to measure the quality of the intervention and to confirm its reproducibility. A SPIRIT checklist has been filled up for this protocol to ensure its standard (Additional file 3).

\section{Benefits and risks of participation}

Every participant will receive a stationery bag as gift containing a notebook, a pencil, an eraser and a sharpener, costing about $\$ 2$, upon completing the baseline survey. No monetary incentive will be provided. 
Any information about the risk behaviors of the individual participants will not be disclosed. Intervention will be given in groups of students where participants possessing all kind of risks will take part instead of separating them in specific group such as physically inactive group or smoker group etc. Thus, we will try to minimize any perceived physical or psycho-social risk for participating in this study. No invasive procedures will be involved.

\section{Ethical consideration}

This study protocol got ethical clearance from Institutional Review Board (IRB) of BSMMU (BSMMU/2018/ 5958). Written permission will be obtained from the authorities of both schools and informed written assent will be collected from every participant prior to participation. Parents or legal guardians of the selected students will be informed about the intervention over telephone and through notice on school diary. If the parents send their children on the day of intervention, it will be regarded as their implied consent [28] to let their children participate in the health education intervention. No written consent will be obtained from the guardians other than the implied consent which has been approved by the IRB, BSMMU.

All the physical measurement will be taken in a separate room maintaining privacy and confidentiality. Female volunteer medical students will measure blood pressure and anthropometric measurements of the female participants.

\section{Reporting of the result}

It is decided to disseminate this intervention and the findings of the proposed study through a dissemination seminar and through reporting on it. The Transparent Reporting of Evaluations with Non-randomized Designs (TREND) statement checklist will be followed while reporting this study to ensure maintaining highest standard and getting maximum possible benefit out of this study in creating evidences.

\section{The intervention}

Potential candidates for intervention will be divided into several groups based on their roll numbers. Each group will contain no more than 25 students. Then the intervention will be delivered to each group through health promotion sessions.

The intervention has been developed with the help of a psychologist, a nutritionist, a child health specialist and some public health specialists having experiences to work with adolescent health. Content of the intervention will be further improved and customized after baseline survey addressing the barriers to healthy behaviors among the target population.

The conceptual framework of the expected change through this intervention is as follows:

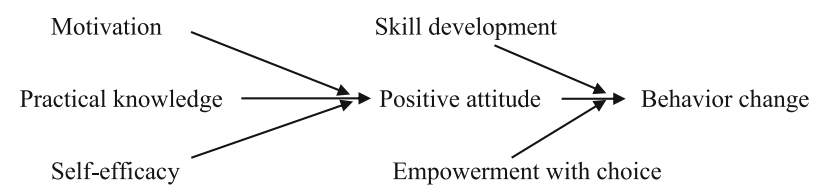

Intervention will be given on the following issues: Issue 1: Diet

1. Understanding that there is an effect of every food we eat on our body and health besides alleviating our appetite

2. Demonstrating all the foods we eat every day and grouping them according to their nutritional values

3. Understanding the metabolism and function of different food groups- carbohydrate, protein, fruits and vegetables, fries and fast food, soft and energy drinks

4. Understanding and identifying healthy and unhealthy food options depending on its function

5. Understanding and identifying the link between unhealthy food options and NCDs

6. Raising curiosity on what should be the food options to prevent NCDs

Issue 2: Physical activity

1. Demonstrating the concept of physical activity and sedentary behavior

2. Understanding and identifying the link between physical inactivity and NCDs

3. Understanding the benefits of three types of exercise (i.e., aerobic, strength building and stretching)

4. Raising curiosity on how much and what type of physical activity should an adolescent do to prevent NCDs

Issue 3: Obesity

1. Demonstrating the concept of normal weight and obesity

2. Enabling to calculate one's Body Mass Index (BMI) status

3. Understanding and identifying the potential causes of obesity

4. Understanding the health consequence of childhood obesity

5. Raising curiosity on what should be the ideal weight to stay fit and how to achieve it 
Issue 4: Smoking

1. Understanding the concept and the effect of active and passive smoking

2. Understanding the relation of smoking with NCDs

3. Identifying the common reasons to start smoking at adolescent age

4. Raising curiosity on how to avoid starting smoking or how to quit smoking or how to reduce passive smoking

Issue 5: NCDs

1. Understanding the basic pathology and aetiology of NCDs with special emphasis on atherosclerosis, stroke, heart disease, diabetes and hypertension

2. Understanding and identifying the effect of lifestyle behavior on NCDs

3. Recognizing the preventive ways of NCDs

4. Raising curiosity on how can and to what extent should one apply the preventive ways to avert NCDs

Issue 6: Preventive ways

1. Understanding the daily requirements of different foods to prevent NCDs

2. Enabling to make one's own platter

3. Understanding the recommended physical activity requirements at adolescent age

4. Enabling to perform aerobic and stretching exercise

5. Understanding the ways to maintain normal body weight

6. Making healthy choices for lifestyle behavior and setting goals

The intervention will be delivered through a health promotion session using multi-media projector, flip paper and pen and leaflets. Each session will be of $2 \mathrm{~h}$ duration. A sequential framework of the intervention conceptualizing from a standard MI training manual [29] and SCT theory [18] will be used to train the facilitators.

Here is the sequential framework of the intervention workout:

\begin{tabular}{lll}
\hline Concept & Objectives & $\begin{array}{l}\text { Illustration } \\
\text { with example }\end{array}$ \\
\hline $\begin{array}{ll}\text { Creation of motivational } \\
\text { environment }\end{array}$ & $\checkmark$ Creating a & . Facilitators will \\
& collaborative & be trained to be \\
& environment that & supportive, \\
& evoke motivation. & suspend an \\
& $\checkmark$ Giving honour & authoritarian role, \\
& to students' autonomy & explore capacity \\
& and self-direction. & of the students
\end{tabular}

Methodology (Continued)

\begin{tabular}{|c|c|c|}
\hline Concept & Objectives & $\begin{array}{l}\text { Illustration } \\
\text { with example }\end{array}$ \\
\hline & & $\begin{array}{l}\text { rather than } \\
\text { incapacity. e.g. } \\
\text { facilitators will } \\
\text { invite the } \\
\text { participants to } \\
\text { correlate different } \\
\text { types of foods } \\
\text { with different } \\
\text { health status in } \\
\text { pictorial view. } \\
\text { Here facilitators } \\
\text { will show a sincere } \\
\text { interest in the } \\
\text { students' } \\
\text { experiences and } \\
\text { perspectives. }\end{array}$ \\
\hline $\begin{array}{l}\text { Establish rapport } \\
\text { and express } \\
\text { empathy }\end{array}$ & $\begin{array}{l}\text { Explaining the } \\
\text { aim of the } \\
\text { session. Showing } \\
\text { concern about } \\
\text { their health. } \\
\text { Making positive } \\
\text { environment } \\
\text { where the } \\
\text { students feel } \\
\text { free to talk } \\
\text { about } \\
\text { themselves. }\end{array}$ & $\begin{array}{l}\text { Facilitators will ask } \\
\text { the students to talk } \\
\text { about their general } \\
\text { perception regarding } \\
\text { some common NCDs } \\
\text { and their risk factors. } \\
\text { Facilitators will also } \\
\text { invite them to share } \\
\text { their experience with } \\
\text { NCD patients in their } \\
\text { family. These } \\
\text { conversations will be } \\
\text { non-judgmental and } \\
\text { with proper empathy. }\end{array}$ \\
\hline $\begin{array}{l}\text { Self-exploration } \\
\text { of behavior }\end{array}$ & $\begin{array}{l}\text { Letting students } \\
\text { explore their } \\
\text { experience, } \\
\text { knowledge and } \\
\text { ambivalence } \\
\text { in a supportive } \\
\text { and facilitative } \\
\text { atmosphere. }\end{array}$ & $\begin{array}{l}\text { - Facilitators will } \\
\text { practice making } \\
\text { open ended } \\
\text { relevant questions, } \\
\text { avoiding } \\
\text { uncomfortable ones, } \\
\text { e.g. facilitators will } \\
\text { ask students about } \\
\text { eating vegetables } \\
\text { regularly. Facilitators } \\
\text { may ask them why } \\
\text { they eat vegetables } \\
\text { every day or why } \\
\text { not, and what will } \\
\text { be the perceived } \\
\text { health } \\
\text { consequences in } \\
\text { both cases without } \\
\text { making any } \\
\text { judgement. The co- } \\
\text { facilitator will jot the } \\
\text { answers briefly } \\
\text { down a flip paper. } \\
\text { Then, facilitators will } \\
\text { summarize } \\
\text { responses and give } \\
\text { affirmation. }\end{array}$ \\
\hline $\begin{array}{l}\text { Practical } \\
\text { knowledge } \\
\text { sharing }\end{array}$ & $\begin{array}{l}\text { Learning by } \\
\text { observation. }\end{array}$ & $\begin{array}{l}\text { Facilitators will show } \\
\text { the link between } \\
\text { sugar sweetened } \\
\text { beverages and } \\
\text { obesity, using pictures } \\
\text { and short videos. } \\
\text { Facilitators will show }\end{array}$ \\
\hline
\end{tabular}


Methodology (Continued)

\begin{tabular}{l} 
Concept \\
\hline Developing \\
discrepancy \\
\\
Recognizing \\
change talk
\end{tabular}

\section{$\checkmark$ Identifying} change talk and commitment language that signals movement in the direction of behaviour change, as well as sustain talk.

Rolling with the conflict of students rather than opposing
Skill development
Developing

Change Plan

\begin{abstract}
Understanding the difference behavior and its consequences health status
\end{abstract}

Roll with resistance

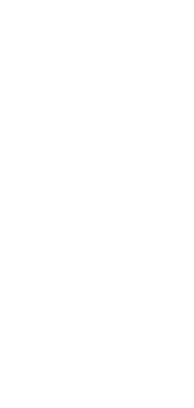

Learning to perform new behaviors to overcome barriers towards healthy lifestyle through observation and peer modeling

\footnotetext{
$\checkmark$ Making the transition into practice.
}

Methodology (Continued)

Illustration

with example

the consequences of different NCDs such as diabetes through videos.

Facilitators will recapitulate their current salty food consumption and help them recognizing the discrepancy between their current behavior and their desire to stay free from cardiovascular diseases.

- Facilitators will try to recognize this kind of talks "maybe my soft drink consumption will put me in trouble" or "now I am worried about my weight" and will give affirmation to these change talk.

Some students may oppose or show disagreement about changing behavior. Facilitators will not compete with them, rather take another approach to make the issue clearer to the students. Facilitators will respond to Students sustain talk and resistance in a manner that reflects respect without reinforcing the desired behavior.

Facilitators will recruit some volunteer role models from the students and teach them how to perform some effective free hand stretching exercises in a resource poor setting. Students will observe the models and adopt the best suit for themselves.

Facilitators will encourage the students to explore their barriers to be

\begin{tabular}{|c|c|c|}
\hline Concept & Objectives & $\begin{array}{l}\text { Illustration } \\
\text { with example }\end{array}$ \\
\hline & & $\begin{array}{l}\text { physically active } \\
\text { and try to discover } \\
\text { their own potential } \\
\text { solutions giving } \\
\text { emphasis on } \\
\text { timing and } \\
\text { negotiation skill. }\end{array}$ \\
\hline $\begin{array}{l}\text { Improve self- } \\
\text { evaluated } \\
\text { outcome } \\
\text { expectancy }\end{array}$ & $\begin{array}{l}\text { Improving hopes } \\
\text { and beliefs about } \\
\text { the consequences } \\
\text { of desired behavior. }\end{array}$ & $\begin{array}{l}\text { Facilitators will help } \\
\text { students evaluate } \\
\text { and improve } \\
\text { expectations about } \\
\text { smoke free life or } \\
\text { physically active life. }\end{array}$ \\
\hline $\begin{array}{l}\text { Consolidating } \\
\text { commitments }\end{array}$ & $\begin{array}{l}\checkmark \text { Eliciting strength } \\
\text { of changing } \\
\text { commitment }\end{array}$ & $\begin{array}{l}\text { - Facilitators will } \\
\text { highlight students' } \\
\text { changing } \\
\text { commitment to } \\
\text { reinforce them. } \\
\text { Facilitators will } \\
\text { give emphasis } \\
\text { on specific } \\
\text { implementation } \\
\text { intentions. }\end{array}$ \\
\hline Support self-efficacy & $\begin{array}{l}\text { Supporting the belief } \\
\text { in personal ability } \\
\text { to adopt healthy } \\
\text { behavior }\end{array}$ & $\begin{array}{l}\text { Students will be } \\
\text { asked to express } \\
\text { their self-efficacy } \\
\text { about doing } \\
\text { physical activity in } \\
\text { an efficacy measuring } \\
\text { scale and write down } \\
\text { individual plan to } \\
\text { acquire adequate } \\
\text { daily physical activity. }\end{array}$ \\
\hline Verbal persuasion & $\begin{array}{l}\text { Strengthening } \\
\text { self-efficacy }\end{array}$ & $\begin{array}{l}\text { Facilitators will } \\
\text { encourage students } \\
\text { with culturally } \\
\text { accepted and } \\
\text { stimulating words } \\
\text { e.g. facilitators may } \\
\text { use trending words } \\
\text { of this age. }\end{array}$ \\
\hline $\begin{array}{l}\text { Setting up self- } \\
\text { regulatory ways }\end{array}$ & $\begin{array}{l}\text { Constructing own } \\
\text { goal and self- } \\
\text { monitoring ways } \\
\text { to achieve the goal }\end{array}$ & $\begin{array}{l}\text { Facilitators will } \\
\text { encourage and } \\
\text { support students } \\
\text { to build personal } \\
\text { goal to consume } \\
\text { at least five servings } \\
\text { of fruits per day and } \\
\text { to set self-monitoring } \\
\text { ways to achieve } \\
\text { the goal. }\end{array}$ \\
\hline
\end{tabular}

\section{Discussion}

This intervention aims to motivate adolescents towards positive health behaviors. Motivation can be postulated as the reason to action. Motivation can change individual's perception and thus prepare oneself to accept the changing process. MI is one of the most thoroughly used and successful method for counseling aimed at promoting health behavior. In this study, students will be motivated using a few MI principles [30, 31] 
to trigger their concern about their own health and then help them going through the changing process. Although MI was designed and used in clinical settings extensively, it can be successfully applied in school settings too [32].

MI is mostly used in alcohol and drug abuse cases [33], and in case of smoking [34]. However, MI is also used in other health behavior changes as well [35]. Originally, MI was designed to be delivered in person but, MI delivered in a group also showed significant success in making desired change [36]. In this study, the principles of MI will be applied to counsel a group of students in school setting, who will be homogenous in terms of age, sex, socio-economic status and risk behavior status. Thus, the true essence of MI principles is expected to be preserved. The facilitators will be selected and trained meticulously on MI, according to the guidelines developed by Miller (1995), to maintain the standard of MI portion of the intervention.

Once the students are motivated enough to show their interest in healthy behavior, the process of negotiating change and strengthening commitment will be started by providing information. The information will be delivered in a structured way based on the principles of Bandura's Social Cognitive Theory (SCT) [12]. The objective is to improve psychological determinants of behavior through observational learning. This intervention will emphasize supporting self-efficacy that is the belief in performance which brings the desire change [13]. As for example, if self-efficacy of a student about healthy diet is high, it means he believes that he can manage healthy eating at any situation. In this intervention, focus will be given on improving this self-efficacy through sharing experience, modeling and through verbal persuasion using some trend words, like "you are Bangladesh, you can do it". In addition, education about NCDs and its risk factors will be given in such a way that allows them to reflect, letting them develop, and evaluate their own outcome expectancy which is, another behavior changing component of Bandura's theory [18].

This intervention is not aimed to bring any environmental change. Yet, this intervention will demonstrate choices and make them skilled, thus empower them to pick the most suitable choice for themselves. As for example, they will be taught with a range of free hand exercises by role modeling and by making some role models among themselves. Now as they are mastered on several exercises, they can do any of them at their convenience, to acquire the daily physical activity recommendation. Leaflets on healthy diet, physical activity and anti-tobacco message will be provided to take home, which is expected to improve the social support to some extent. These will eventually improve their self-efficacy and self-regulatory capacity and thus influence health behavior.

An intervention is judged by its cost-effectiveness when referring for mass population. Meta-analysis on this issue found that group intervention is the least expensive yet most cost-effective interventional way among the adolescents [37]. This proposed intervention is designed for group and therefore, is more realistic in the low socio-economic context like Bangladesh to deliver the intervention to utilize the limited resources best. Thus, upon its effectiveness being proven, this intervention can be advocated to policies.

Any behavior to persist long-term requires social support, supportive environments, and periodic reinforcement [18]. Social engagement or environmental reformation is beyond the scope of this study and the long-term reinforcement of the student. With limited time and financial support, present study is expected to gather valuable knowledge about development of effective NCDs prevention intervention among adolescents of Bangladesh.

\section{Additional files}

Additional file 1: Study design (Stages of the study) (DOC $33 \mathrm{~kb}$ )

Additional file 2: Questionnaire (in English) (DOC 253 kb)

Additional file 3: SPIRIT Checklist (DOC $131 \mathrm{~kb}$ )

Abbreviations

BMI: Body mass index; BSMMU: Bangabandhu Sheikh Mujib Medical University; Ml: Motivational interview; NCD: Non-communicable disease;

SCT: Social cognitive theory; SSB: Sugar sweetened beverage; STEPS: Stepwise Surveillance for NCD risk factors; TREND: Transparent reporting of evaluations with non-randomized designs

\section{Acknowledgements}

Authors acknowledged SM Atiqur Rahman, Consultant, Department of Psychiatrics, BSMMU; Mohammad. Tanvir Islam, Associate Professor, Department of Internal Medicine, BSMMU and Jahanara Akter Sumi, Nutritionist, BSMMU for their valuable contribution in developing the intervention.

\section{Authors' contributions}

MS developed the protocol and the intervention and wrote the main manuscript; MAH developed protocol and the intervention; MK is the principal investigator and contributed in protocol and intervention development; MAAM, MRB contributed in development of the intervention and manuscript writing, and SRC contributed in development of the protocol and the intervention. All the authors read and approved the manuscript for submission.

\section{Funding}

This study received financial assistance from Directorate General of Health Services, Ministry of Health \& Family Welfare, Mohakhali, Dhaka-1212 (DGHS/ LD/NCDC/Procurement Plan/GOB (Service)/2017-2018/539/SP-06 Dated:

13.11.2017). This protocol has been peer-reviewed by the Directorate General of Health Services, Ministry of Health \& Family Welfare, Bangladesh before selecting for financial assistance. The funding body has no influence or control over study conduction including manpower recruitment, field data collection, intervention, data analysis and interpretation. Writing manuscript on study protocol is also independent of the funding body.

Availability of data and materials

Data sharing is not applicable to this article as this is a protocol paper. 


\section{Ethics approval and consent to participate}

Ethical approval of this protocol was obtained from BSMMU's Institutional Review Board (BSMMU/2018/5958). Informed written consent will be obtained from school authority and the principal and informed written assent will be obtained from every student before participation in the study. Consent from the parents or legal guardians of the students will be obtained through implied consent procedure following WHO guideline. Parents or legal guardians of the selected students will be informed about the intervention over telephone and through notice on school diary. If the parents send their children on the day of intervention, it will be regarded as their implied consent. No written consent will be obtained from the guardians other than the implied consent as only a few students will be invited to join the health education program, and this procedure of taking implied consent has been approved by the Institutional Review Board, Bangabandhu Sheikh Mujib Medical University.

\section{Consent for publication}

Not applicable.

\section{Competing interests}

The authors declare that they have no competing interests.

\section{Author details}

${ }^{1}$ Chi Research \& Infotec Ltd, Dhaka, Bangladesh. ${ }^{2}$ Department of Public Health and Informatics, Bangabandhu Sheikh Mujib Medical University, Dhaka, Bangladesh. ${ }^{3}$ Department of Epidemiology \& Research, National Heart Foundation Hospital \& Research Institute, Dhaka, Bangladesh.

\section{Received: 19 May 2019 Accepted: 24 June 2019}

Published online: 25 July 2019

\section{References}

1. Diez-Canseco F, Boeren Y, Quispe R, lin CM, Miranda JJ. Engagement of adolescents in a health communications program to prevent noncommunicable diseases: Multiplicadores Jóvenes, Lima, Peru, 2011. Prev Chronic Dis. 2015;12:140416.

2. Sawyer SM, Afi RA, Bearinger LH, Blakemore S, Dick B, Ezeh AC, et al. Adolescent health 1 adolescence : a foundation for future health. Lancet. 2012;379:1630-40

3. British Medical Association. Exploring the cost effectiveness of early intervention and prevention. 2017. https://www.bma.org.uk/-/media/files/pdfs/collective\%20 voice/policy\%20research/public\%20and\%20population\%20health/exploring-thecost-of-early-intervention-ill-health-prevention.pdf?la=en. Accessed 5 July 2019.

4. Döring N, Mayer S, Rasmussen F, Sonntag D. Economic evaluation of obesity prevention in early childhood: methods, limitations and recommendations. Int J Environ Res Public Health. 2016;13(9):1-11.

5. Bhuiyan MU, Zaman S, Ahmed T. Risk factors associated with overweight and obesity among urban school children and adolescents in Bangladesh: A case-control study. BMC Pediatr. 2013;13:72.

6. GAIN. Nutrition in adolescence: Bangladesh. Global Alliance for improved nutrition. 2018. https://www.gainhealth.org/wp-content/uploads/2018/06/ Adolescent-Nutrition-_-Bangladesh-2018.pdf. Accessed 12 Jan 2019.

7. Uddin R, Khan A, Burton NW. Prevalence and sociodemographic patterns of physical activity among Bangladeshi young adults. J Health Popul Nutr. 2017;36(1):31.

8. Khan MM, Karim MR, Alam MS, Ali MM, Masud J. Prevalence and determinants of smoking among adolescent boys in Dhaka City. Anwer Khan Modern Med Coll J. 2018;9(1):34-8. https://doi.org/10.3329/akmmcj.v9i1.35822

9. De $\mathrm{VH}$, Kok C. From determinants of smoking behaviour to the implications for a prevention programme. Health Educ Res. 1986;1(2):85-94. https://doi. org/10.1093/her/1.2.85.

10. Kim M-S, Hunter JE. Relationships among attitudes, behavioral intentions, and behavior: A meta-analysis of past research, part 2. Communic Res. 1993;20(3).

11. Evans G, Durant J. The relationship between knowledge and attitudes in the public understanding of science in Britain. Public Underst Sci. 1995;4(1):57-74.

12. Bandura A. Social cognitive theory : an agentic perspective. Asian J Soc Psychol. 1999;2(1):21-41.

13. Bandura A. Self-efficacy: toward a unifying theory of behavioral change. Adv Behav Res Ther. 1977;84(2):191-215. https://doi.org/10.1016/0146-6402(78)90002-4.
14. De Vries H, Kijkstra MKP. Self-efficacy: the triad factor resides attitude and subjective norm as a predictor of behavioral intentions. Health Educ Res. 1998;3(3):273-82.

15. Bandura A, Adams NE. Analysis of self-efficacy theory of behavioral change. Cognit Ther Res. 1977;1(4):287-8.

16. Naar-king S, Suarez M. Motivational interviewing with adolescents and young adults. 1st ed. New York: Guilford Press; 2010.

17. Rollnick S, Miller WR. What is motivational interviewing ? Behav Cogn Psychother. 1995;23(4):325-34.

18. Glanz K, Rimer BK, Viswanath K. Health behavior and health education theory, research, and practice. 4th ed. San Francisco: Jossey-Bass: A Wiley Imprint; 2008.

19. Bhattacharyya O, Reeves S, Garfinkel S, Zwarenstein M. Designing theoreticallyinformed implementation interventions: fine in theory, but evidence of effectiveness in practice is needed. Implement Sci. 2006;1 (1):3-5.

20. Rohrbach LA, Grana R, Sussman S, Valente TW. Type II translation: transporting prevention interventions from research to real-world settings. Eval Heal Prof. 2006;29(3):302-33.

21. Nigg CR. Theory-comparison and multiple-behavior research: common themes advancing health behavior research. Health Educ Res. 2002;17(5):670-9.

22. Goldstein MG, Whitlock EP, DePue J. Multiple behavioral risk factor interventions in primary care. Am J Prev Med. 2004;27(2):61-79.

23. Gourlan M, Sarrazin P, Trouilloud D. Motivational interviewing to promote physical activity in obese adolescents: A randomized-controlled trial using self-determination theory as an explanatory framework. Psychol Health. 2013:28(11):1265-86. https://doi.org/10.1080/08870446.2013.800518.

24. WHO. Global School-based Student Health Survey Bangladesh. 2014. http:// www.who.int/chp/gshs/bangladesh/en/. Accessed 10 Jan 2019.

25. Directorate general of health services Ministry of Health and Family Welfare. Non-communicable disease risk factor survey Bangladesh 2010. World Health Organization - Country Office for Bangladesh. 2011. http://www. searo.who.int/entity/noncommunicable_diseases/data/Bangladesh_2010_ STEPS_Survey_Report.pdf. Accessed 30 Dec 2018.

26. Demaio AR, Dugee O, Amgalan G, Maximenco E, Munkhtaivan A, Graeser S, et al. Protocol for a national, mixed-methods knowledge, attitudes and practices survey on non-communicable diseases. BMC Public Health. 2011;11(1):961.

27. WHO. Waist circumference and waist-hip ratio: report of a $\mathrm{WHO}$ expert consultation. Geneva: World Health Organization (WHO); 2008.

28. WHO. Considerations regarding consent in vaccinating children and adolescents between 6 and 17 years old. Geneva: World Health Organization; 2014. http://www.who.int/immunization/programmes_ systems/policies_strategies/consent_note_en.pdf. Accessed 10 Jul 2018

29. MINT. Motivational Interviewing Training New Trainers Manual. Vol. 2, Motivational Interviewing Network of Trainers (MINT). 2014. http://www. motivationalinterviewing.org/sites/default/files/tnt_manual_2014_d10_ 20150205.pdf. Accessed 13 Jan 2019.

30. Miller WR, Moyers TB. Eight stages in learning motivational interviewing. J Teach Addict. 2006;5(1):3-17.

31. Miller WR, Rollnick S. Motivational interviewing: helping people change. 3rd ed. New York: Guilford Publications; 2013.

32. Grenard JL, A M, Ames SL, Ph D, Wiers RW, Ph D, et al. Brief Intervention for Substance Use among At-Risk Adolescents : A Pilot Study. 2007:40:188-191.

33. Spirito A, Monti PM, Barnett NP, Colby SM, Sindelar H, Rohsenow DJ, William Lewander MM. A randomized clinical trial of a brief motivational intervention for alcohol-positive adolescents treated in an emergency department. J Pediatr. 2004;145(3):396-402.

34. Lawendowski LA. A motivational intervention for adolescent smokers. Prev Med. 1998;46(27):39-46. https://doi.org/10.1006/pmed.1998.0424.

35. Stevens VJ, Brown KM, Van HL, Gernhofer N, Peters E, Greenberg R, et al. A brief motivational intervention to improve dietary adherence in adolescents. Health Educ Res. 1999;14(3):399-410. https://doi.org/10.1093/her/14.3.399.

36. D'Amico EJ, Osilla KC, Hunter SB. Developing a group motivational interviewing intervention for first-time adolescent offenders at-risk for an alcohol or drug use disorder. Alcohol Treat Q. 2010;28(4):417-36.

37. French MT, Ph D, Zavala SK, H MP, Mccollister KE, Ph D, et al. Cost-effectiveness analysis of four interventions for adolescents with a substance use disorder. J Subst Abuse Treat. 2008;34:272-81. https://doi.org/10.1016/j.jsat.2007.04.008.

\section{Publisher's Note}

Springer Nature remains neutral with regard to jurisdictional claims in published maps and institutional affiliations. 